\title{
Simple and efficient method for protection of hydroxyl groups as 4- methoxybenzyl ethers
}

\author{
Anna Kasprzycka ${ }^{1}$, Agata Ptaszek-Budniok and Wiesław Szeja \\ Silesian Technical University, Department of Chemistry, ul. Krzywoustego 4,44-100 Gliwice, Poland
}

\section{SUPPORTING INFORMATION}

\section{General procedure for the preparation of 4-Methoxybenzyl N-allyl}

\section{thiocarbamate (2) (Scheme 1)}

4-Methoxybenzyl N-allyl thiocarbamate (2) was prepared according to procedure of alcohol thiocarbamates preparation in microwave reactor. A solution of corresponding alcohol (138 $\mathrm{mg}, 1 \mathrm{mmol}$ ) in toluene was stirred at room temperature for $20 \mathrm{~min}$ in the presence of $4 \AA$ micronized molecular sieves $(10 \mathrm{mg})$. Suitable base (Table 1) and $\mathrm{N}$-allyl isothiocyjanate (0.39. $\mathrm{ml}, 4 \mathrm{mmol})$ were added and subsequently reaction mixture was exposed to microware irradiation $(2 \mathrm{~W})$ for $1 \mathrm{~h}$ at a temperature of $50^{\circ} \mathrm{C}$. Reaction progress was monitored by TLC (toluene/EtOAc 8/1). Afterward, the reaction mixture was filtered and washed with brine $(2 \times 3$ $\mathrm{ml})$, dried $\left(\mathrm{MgSO}_{4}\right)$ and the solvent was evaporated. The crude product was purified by column chromatography on silica gel eluting EtOAc/hexane (1/18). Fractions containing the product were combined, and solvent was removed to afford corresponding alcohol $\mathrm{N}$-allyl thiocarbamate.

\footnotetext{
${ }^{1}$ Corresponding author., e-mail: anna.kasprzycka@polsl.pl
} 


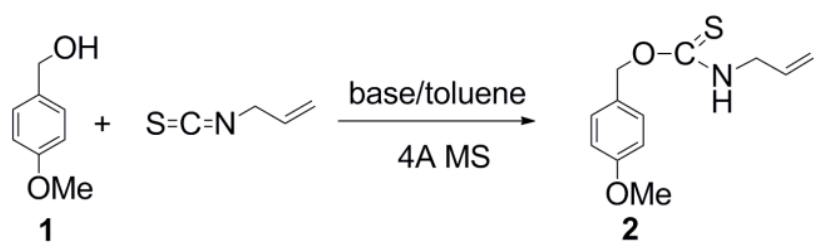

Scheme 1.

Table 1. Suitable base using to synthesis of alcohol N-allyl-thiocarbamate

\begin{tabular}{lll}
\hline Entry & Base & $\begin{array}{l}\text { Equivalent } \\
(\mathrm{mmol})\end{array}$ \\
\hline 1. & $\mathrm{~K}_{2} \mathrm{CO}_{3}$ & 1.5 \\
2. & $\mathrm{iPr}_{2} \mathrm{NEt}$ & 1 \\
3. & $\mathrm{BEMP}$ & 0.01 \\
4. & $\mathrm{DBU}$ & 0.15 \\
\hline
\end{tabular}

4-Methoxybenzyl N-allyl thiocarbamate (2) Yield: 94\%; yellow oil; 7.29-7.33 (2H, m, o$\underline{\mathrm{Ph}}), 6.87$ - $6.91(2 \mathrm{H}, \mathrm{m}, m-\underline{\mathrm{Ph}}), 6.40(1 \mathrm{H}, \mathrm{br} . \mathrm{s}, \underline{\mathrm{NH}}), 5.80-5.91(1 \mathrm{H}, 2 \mathrm{dt}, J 10.2 \mathrm{~Hz}, J 17.1 \mathrm{~Hz}, J$ $\left.5.8 \mathrm{~Hz}^{1}{ }^{\prime} \mathrm{CH}_{2}{ }^{2} \underline{\mathrm{CH}}^{3} \mathrm{CH}_{2}\right), 5.45\left(2 \mathrm{H}, \mathrm{ps}, \underline{\mathrm{CH}}_{2} \mathrm{Ph}\right), 5.15-5.20\left(2 \mathrm{H}, \mathrm{m},{ }^{1} \mathrm{CH}_{2}{ }^{2} \mathrm{CH}^{3} \underline{\mathrm{CH}}_{2}\right), 4.17-$ $4.21\left(2 \mathrm{H}, \mathrm{tt}, J 5.8 \mathrm{~Hz}, J 3.05 \mathrm{~Hz}, J 1.52 \mathrm{~Hz},{ }^{1} \underline{\mathrm{CH}}_{2}{ }^{2}{ }^{\prime} \mathrm{CH}^{3}{ }^{3} \mathrm{CH}_{2}\right), 3.80(3 \mathrm{H}, \mathrm{s}, \mathrm{PhOMe}) ; \mathrm{d}_{\mathrm{C}}(100.6$ $\left.\mathrm{MHz}, \mathrm{CDCl}_{3}\right)$ 190.12, 159.60, 132.48, 130.18, 128.66, 117.43, 113.80, 71.82, 55.17, 47.54; HRMS (ESI- MS): calcd for $\mathrm{C}_{12} \mathrm{H}_{15} \mathrm{NO}_{2} \mathrm{~S}: 260.0721[\mathrm{M}+\mathrm{Na}]^{+}$; found: 260.0709 . 


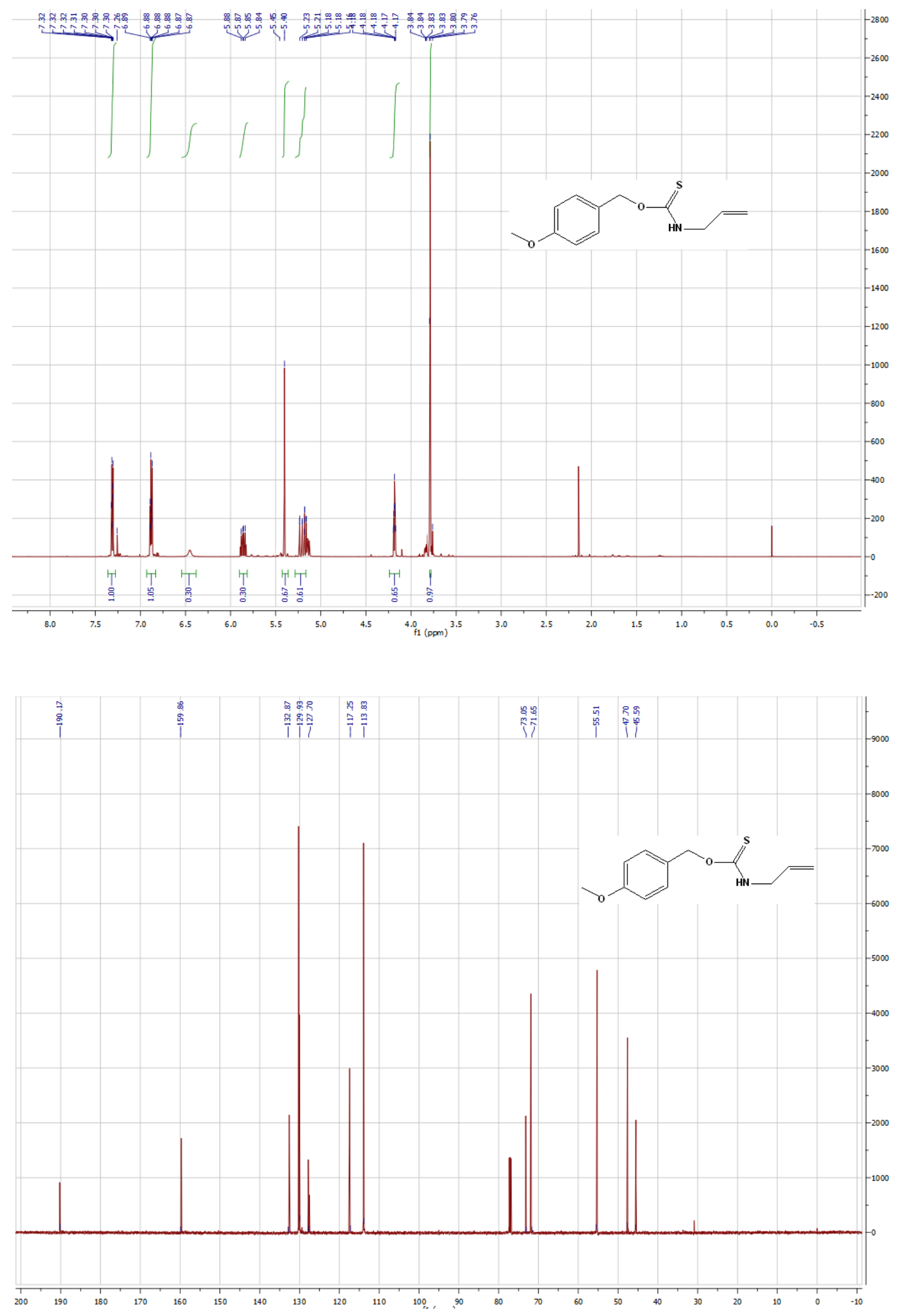




\section{General procedure for the Reaction of various types of alcohols (Scheme 2)}

A solution of alcohol $(1 \mathrm{mmol})$ in methyl chloride $(1.5 \mathrm{ml})$ was stirred at room temperature for $20 \mathrm{~min}$. in the presence of molecular sieves (4 $\AA, 50 \mathrm{mg}$ ). After $20 \mathrm{~min}$. the mixture was stirred at $-25^{\circ} \mathrm{C}$ temperature for $10 \mathrm{~min}$. 4- methoxybenzyl $\mathrm{N}$-allyl thiocarbamate (2 mmol) in methylene chloride $(1.5 \mathrm{ml})$ was stirred in second flask at $-25{ }^{0} \mathrm{C} . \mathrm{N}$ Bromosuccinimide (NBS) (2 mmol ) and $\mathrm{Et}_{4} \mathrm{NTfl}(2 \mathrm{mmol})$ were dissolved in methyl chloride $(1.5 \mathrm{ml})$ were in third flask and this mixture was also cooled down at $-25^{\circ} \mathrm{C}$. Cooled flasks were then connected and $\mathrm{TfOH}(0,01 \mathrm{mmol})$ in methylene chloride $(0,5 \mathrm{ml})$ was added to the mixture. The reaction was completed (TLC, 3:1 hexane/ethyl acetate) in 6 minutes. The reaction mixture was neutralized with $\mathrm{Et}_{3} \mathrm{~N}(0,05 \mathrm{mmol})$, filtered and washed with brine (1x3ml). The solution was dried $\left(\mathrm{MgSO}_{4}\right)$ and evaporated. The crude product was purified by column chromatography (hexane/ethyl acetate $25 / 1 \mathrm{v} / \mathrm{v}$ ).

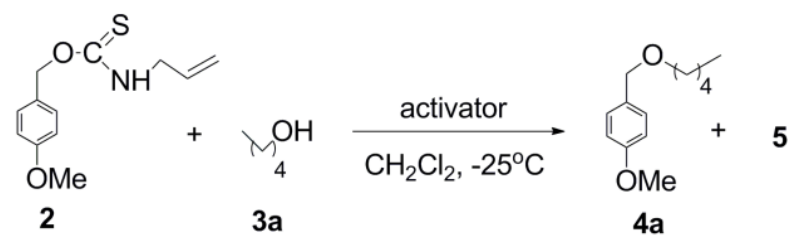

Scheme 2.

1-Methoxy-4-(pentyloxy)benzene (4a) ${ }^{\mathbf{1}}$ Yield: $83 \%$; colorless oil; $\mathrm{d}_{\mathrm{H}}\left(400 \mathrm{MHz} \mathrm{CDC}_{3}\right)$ $7.26(2 \mathrm{H}, \mathrm{d}, J 8.3 \mathrm{~Hz}, o-\underline{\mathrm{Ph}}), 6.89(2 \mathrm{H}, \mathrm{d}, J 8.3 \mathrm{~Hz}, m-\underline{\mathrm{Ph}}), 4.33\left(2 \mathrm{H}, \mathrm{s}, \underline{\mathrm{CH}}_{2} \mathrm{Ph}\right) 3.79(3 \mathrm{H}, \mathrm{s}$, $\mathrm{PhOMe}), 3.70\left(2 \mathrm{H}, \mathrm{m}, \mathrm{OC}_{2}{ }_{2}\left(\mathrm{CH}_{2}\right)_{3} \mathrm{Me}\right), 1.59-1.54\left(2 \mathrm{H}, \mathrm{m}, \mathrm{OCH}_{2} \underline{\mathrm{C}}_{2}\left(\mathrm{CH}_{2}\right)_{2} \mathrm{Me}\right), 1.3-1.21$ (4H, m, O $\left(\mathrm{CH}_{2}\right)_{2}\left(\mathrm{C}_{2}{ }_{2}{ }_{2} \mathrm{Me}\right), 0.87-0.84\left(3 \mathrm{H}, \mathrm{m}, \mathrm{O}\left(\mathrm{CH}_{2}\right)_{2}\left(\mathrm{CH}_{2}\right)_{2} \underline{\mathrm{Me}}\right) ; \mathrm{d}_{\mathrm{C}}\left(100.6 \mathrm{MHz}, \mathrm{CDCl}_{3}\right)$ 159.0, 130.7, 129.1, 113.6, 72.4, 70.1, 55.2, 29.4, 28.3, 22.5, 14.0.; HMRS (EI): calcd for $\mathrm{C}_{13} \mathrm{H}_{20} \mathrm{O}: 208.1463[M]^{+}$; found: $208.1457 .{ }^{1} \mathrm{H},{ }^{13} \mathrm{C}$ NMR and HMRS spectra were identical with those in the literature ${ }^{1}$. 
1-(tert-Butoxymethyl)-4-methoxybenzene (4b) ${ }^{\mathbf{1}}$ Yield: $80 \%$; colorless oil; $\mathrm{d}_{\mathrm{H}}(400 \mathrm{MHz}$ $\left.\mathrm{CDC1}_{3}\right) 7.25(2 \mathrm{H}, \mathrm{d}, J 8.3 \mathrm{~Hz}, o-\underline{\mathrm{Ph}}), 6.85(2 \mathrm{H}, \mathrm{d}, J 8.3 \mathrm{~Hz}, m-\underline{\mathrm{Ph}}), 4.38\left(2 \mathrm{H}, \mathrm{s}, \underline{\mathrm{CH}_{2}} \underline{\mathrm{Ph}}\right) 3.79$ (3H, s, PhOMe $), 1.29$ (9H, s , $\mathrm{OC}\left(\underline{\mathrm{CH}_{3}} \underline{2}_{3}\right) ; \mathrm{d}_{\mathrm{C}}\left(100.6 \mathrm{MHz}, \mathrm{CDCl}_{3}\right)$ 158.8, 131.9, 128.9, 113.7, 73.2, 63.7, 55.3, 27.7.; HMRS (EI): calcd for $\mathrm{C}_{12} \mathrm{H}_{18} \mathrm{O}$ : $194.1306[M]^{+}$; found: 194.1297. ${ }^{1} \mathrm{H}$, ${ }^{13} \mathrm{C}$ NMR and HMRS spectra were identical with those in the literature ${ }^{1}$.

Reference:

1. Cuenca, A. B.; Mancha, G.; Asensio, G.; Medio-Simon, M. Chemistry A European Journal "Water Compatible Gold(III)-Catalysed Synthesis of Unsymmetrical Ethers from Alcohols", 2008, 14, 1518.

4-Methoxybenzyl allyl ether (4c) $)^{2}$ Yield: $70 \%$; colorless oil; $\mathrm{d}_{\mathrm{H}}\left(400 \mathrm{MHz} \mathrm{CDC1}_{3}\right) 7.27(2 \mathrm{H}$, $\left.\mathrm{d}, J 8.7 \mathrm{~Hz}, o_{-} \underline{\mathrm{Ph}}\right), 6.87(2 \mathrm{H}, \mathrm{d}, J 8.7 \mathrm{~Hz},-m-\underline{\mathrm{Ph}}), 5.95-5,90\left(1 \mathrm{H}, \mathrm{m}, \mathrm{OCH}_{2} \underline{\mathrm{CHCH}_{2}}\right) 5.32-5.18$ $\left(2 \mathrm{H}, \mathrm{m}, \mathrm{OCH}_{2} \mathrm{CHCH}_{2}\right), 4.54\left(2 \mathrm{H}, \mathrm{s}, \underline{\mathrm{CH}}_{2} \mathrm{Ph}\right), 4.01\left(2 \mathrm{H}, \mathrm{d}, J 5.7 \mathrm{~Hz}, \mathrm{OC}_{2} \underline{\mathrm{CHCH}}_{2}\right), 3.81(3 \mathrm{H}$, s PhOMe); $\mathrm{d}_{\mathrm{C}}\left(100.6 \mathrm{MHz}, \mathrm{CDCl}_{3}\right) 159.10,134.79,130.30,129.23,116.86,113.67,71.67$, 70.75, 55.12.; HRMS (EI): calcd for $\mathrm{C}_{11} \mathrm{H}_{14} \mathrm{O}_{2}\left[\mathrm{M}^{+}\right]$178.0994; found 178.0993. ${ }^{1} \mathrm{H},{ }^{13} \mathrm{C}$ NMR and HMRS spectra were identical with those in the literature ${ }^{2}$.

References:

2. Harada, N.; Nishikata, T.; Nagashima, H. Tetrahedron, ,, Vinyl polymerization versus [1,3] O to C rearrangement in the ruthenium-catalyzed reactions of vinyl ethers with hydrosilanes" 2012, 68, 3243

Cholesteryl (4-methoxybenzyl) ether (4d) ${ }^{3}$ Yield: $80 \%$; colorless oil $\mathrm{d}_{\mathrm{H}}\left(400 \mathrm{MHz} \mathrm{CDC1}_{3}\right)$ $7.26\left(2 \mathrm{H}, \mathrm{d}, J 8.4 \mathrm{~Hz}, o_{-} \underline{\mathrm{Ph}}\right), 6.86(2 \mathrm{H}, \mathrm{d}, J 8.4 \mathrm{~Hz}, m-\underline{\mathrm{Ph}}), 4.49\left(2 \mathrm{H}, \mathrm{s}, \underline{\mathrm{CH}}_{2} \mathrm{Ph}\right), 3.80(3 \mathrm{H}, \mathrm{s}$ $\mathrm{PhOMe}), 3.42-3.50(2 \mathrm{H}, \mathrm{m}$, cholesterol $), 1.40-2.40(21 \mathrm{H}, \mathrm{m}$, cholesterol $), 1.33-1.38(3 \mathrm{H}$, m, cholesterol), $1.10-1.30$ (6H, m, cholesterol), 0.99 (3H, s, cholesterol), $0.92-0.98(2 \mathrm{H}, \mathrm{m}$, cholesterol), $0.87\left(3 \mathrm{H}, \mathrm{s}\right.$, cholesterol), $0.67\left(3 \mathrm{H}, \mathrm{s}\right.$, cholesterol); $\mathrm{d}_{\mathrm{C}}\left(100.6 \mathrm{MHz}, \mathrm{CDCl}_{3}\right)$ 
159.0, 141.0, 131.1, 129.2 (2C), 121.5, 113.8 (2C), 78.3, 69.6, 56.8, 56.2, 55.3, 50.2,42.3, $39.8,39.5,39.2,37.3,36.9,36.2,35.8,32.0,31.9,28.5,28.3,28.0,24.3,23.8,22.8,22.6,21.1$, 19.4, 18.7, 11.9. ${ }^{1} \mathrm{H}$ and ${ }^{13} \mathrm{C}$ NMR spectra were identical with those in the literature ${ }^{3}$.

References:

3. Jung, M. E.; Koch, P. Tetrahedron Letters, ” Mild, selective deprotection of PMB ethers with triflic acid/1,3-dimethoxybenzene”, 2011, 52, 6051.

( \pm )-2-(4-Methoxyphenylmethoxymethyl) oxirane $(4 \mathbf{e})^{4}$ Yield: $81 \%$; colorless oil; $\mathrm{d}_{\mathrm{H}}(400$ $\left.\mathrm{MHz} \mathrm{CDC1}_{3}\right) 7.27(2 \mathrm{H}, \mathrm{d}, J 8.3 \mathrm{~Hz}, o-\underline{\mathrm{Ph}}), 6.87(2 \mathrm{H}, \mathrm{d}, J 8.3 \mathrm{~Hz}, m-\underline{\mathrm{Ph}}), 4.52(2 \mathrm{H}, \mathrm{dd}, J 11.7$ $\left.\mathrm{Hz}, J 7.81 \mathrm{~Hz}, \underline{\mathrm{CH}_{2}} \mathrm{Ph}\right), 3.82(3 \mathrm{H}, \mathrm{s} \quad \mathrm{PhOMe}), 3.72(1 \mathrm{H}, \mathrm{dd}, J 11.48 \mathrm{~Hz}, J 2.93$ $\left.\mathrm{Hz}, \mathrm{CH}_{2} \mathrm{OCHCH}_{2}\right), 3.41\left(1 \mathrm{H}, \mathrm{dd}, J 11.48 \mathrm{~Hz} J 5.86 \mathrm{~Hz}, \mathrm{CH}_{2} \mathrm{OCHCH}_{2} \underline{\mathrm{O}}\right), 3.20-3.15(1 \mathrm{H}, \mathrm{m}$ $\left.\mathrm{CH}_{2} \mathrm{OCHCH}_{2} \mathrm{O}\right), 2.81\left(1 \mathrm{H}, \mathrm{t}, J 4.39 \mathrm{~Hz}, J 9.28 \mathrm{~Hz} \underline{\mathrm{CH}}_{2} \mathrm{OCHCH}_{2} \mathrm{O}\right), 2.61(1 \mathrm{H}, \mathrm{m}$, $\left.\underline{\mathrm{CH}}_{2} \mathrm{OCHCH}_{2} \mathrm{O}\right) ; \mathrm{d}_{\mathrm{C}}\left(100.6 \mathrm{MHz}, \mathrm{CDCl}_{3}\right)$ 159.1, 129.8, 129.3, 113.6, 72.8, 70.4, 55.1, 50.7, 44.; MS m/z (EI) 194 ([M] $\left.]^{+}, 100 \%\right), 163$ ([M-OMe $\left.]^{+}, 6\right), 122$ (69), 78 (22); Found: 194.0950, $\left[\mathrm{C}_{11} \mathrm{H}_{14} \mathrm{O}_{3}\right]^{+}$requires 194.0943. ${ }^{1} \mathrm{H},{ }^{13} \mathrm{C}$ NMR and HMRS spectra were identical with those in the literature ${ }^{4}$.

References:

4. Walker, Louise F.; Bourghida, A.; Connolly, S.; Wills, M. Journal of the Chemical Society, Perkin Transactions I, "Synthesis of 2,5-dihydrofurans via alkylidene carbene insertion reactions, $\mathbf{2 0 0 2}, 965$.

1,2:5,6-Di-O-isopropylidene-3-O-(4-methoxybenzyl)- $\alpha$-D-glucofuranose (4f) Yield: $78 \% \mathrm{~d}_{\mathrm{H}}$ $\left(400 \mathrm{MHz} \mathrm{CDC1}_{3}\right) 7.28(2 \mathrm{H}, \mathrm{dd}, J 6.8 \mathrm{~Hz} o-\underline{\mathrm{Ph}}), 6.88(2 \mathrm{H}, \mathrm{dd}, J 6 \mathrm{~Hz}, m-\underline{\mathrm{Ph}})$, ), $5.89(1 \mathrm{H}, \mathrm{d}$,

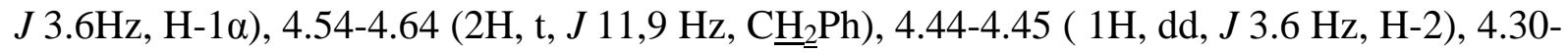
4.40 (2H, m, H-3, H-5), 3.98-4.18 (3H, m, H-4, H-6, H-6), 3.82 (3H, s, PhOMe), 1.32, 1.39, 
1.50, $1.58\left(12 \mathrm{H}, 4 \mathrm{~s}, 2 \mathrm{x}\left(\mathrm{CH}_{3}\right)_{2} \mathrm{C}\right) ; \mathrm{d}_{\mathrm{C}}\left(100.6 \mathrm{MHz}, \mathrm{CDCl}_{3}\right)$ 159.4, 129.7, 129.3, 113.8, 111.8, 108.9, 105.3, 82.7, 81.3(3), 81.3(1), 73.0, 72.1, 67.4, 55.3, 26.8(4), 26.7(7), 26.3, 25.5.; HRMS (EI): m/z: calcd for $\mathrm{C}_{20} \mathrm{H}_{28} \mathrm{O}_{7}: 380.1835$, found $380.1829[\mathrm{M}+] .{ }^{1} \mathrm{H},{ }^{13} \mathrm{C}$ NMR and HMRS spectra were identical with those in the literature. ${ }^{5,6}$

References:

5. Dang, H.-S.; Roberts B. P. Journal of the Chemical Society, Perkin Transactions I, ” Deoxygenation of tertiary and secondary alcohols $\mathrm{ROH}$ by thiol-catalysed radicalchain redox decomposition of derivatives $\mathrm{ROCH}_{2} \mathrm{X}$ to give $\mathrm{RH}$ and $\mathrm{XCHO}$ ” 2002 , 1161.

6. Kurita, T.; Hattori, K.; Seki, S.; Mizumoto, T.; Aoki, F.; Yamada, Y.; Ikawa, K.; Maegawa, T.; Monguchi,Y.; Sajiki, H.; Chemistry A European Journal, ” Efficient and Convenient Heterogeneous Palladium-Catalyzed Regioselective Deuteration at the Benzylic Position”, 2008, 14, 664.

1,2:3,4-Di-O-isopropylidene-6-O-(4-metoksybenzylo)- $\alpha$-D-galactopyranose $(4 \mathrm{~g}){ }^{7}$ Yield: $81 \mathrm{~d}_{\mathrm{H}}\left(400 \mathrm{MHz} \mathrm{CDC1}_{3}\right) 7.26(2 \mathrm{H}, \mathrm{dd},, J 6.2 \mathrm{~Hz} o-\underline{\mathrm{PhOMe}}), 6.86(2 \mathrm{H}, \mathrm{dd}, J 6.2 \mathrm{~Hz} \mathrm{~m}-$ $\underline{\mathrm{PhOMe}}$ ), $5.54(1 \mathrm{H}, \mathrm{d}, J 3.5 \mathrm{~Hz}, \mathrm{H}-1), 4.58(1 \mathrm{H}, \mathrm{dd}, J 3.5 \mathrm{~Hz}, J 2.2 \mathrm{~Hz}, \mathrm{H}-3), 4.49$ (2H, dd, J $\left.12 \mathrm{~Hz}, \underline{\mathrm{C}}_{2} \mathrm{Ph}\right), 4.30-4.32$ (1H, dd, J $\left.2.2 \mathrm{~Hz}, \mathrm{H}-2\right), 4.25-4.29$ (1H, m, H-4), 3.90-4.00 (1H m, , H-5), $3.80(3 \mathrm{H}, \mathrm{s}, \mathrm{OMe}), 3.57-3.66$ (2H, m, H-6, H-6), 1.33, 1.34, 1.44, $1.53(12 \mathrm{H}, 4 \mathrm{~s}$, $\left.2 \mathrm{x}(\underline{\mathrm{Me}})_{2} \mathrm{C}\right) ; \mathrm{d}_{\mathrm{C}}\left(100.6 \mathrm{MHz}, \mathrm{CDCl}_{3}\right) 159.2,131.3,130.5,129.4,114.4,113.8,109.2,96.4$, 73.0, 71.2, 70.6, 68.6, 66.9, 55.3, 26.0, 24.5.; HRMS: calcd for $\mathrm{C}_{20} \mathrm{H}_{27} \mathrm{O}_{7}$ 379.175679, found 379.176152. ${ }^{1} \mathrm{H},{ }^{13} \mathrm{C}$ NMR and HMRS spectra were identical with those in the literature ${ }^{7}$.

Reference: 
7. Sharma, G. V. M.; Mahalingam, A.K. J. Org. Chem.” A Facile Conversion of Alcohols into p-Methoxybenzyl Ethers (PMB-ethers) Using p-Methoxybenzyl Alcohol-Yb(OTf)3”, 1999, 64, 8943.

Methyl-2,3,4-tri-O-benzoilo-6-O-(4-metoksybenzylo)- $\alpha$-D-glucopyranoside $(4 \mathrm{~h})^{8}$ Yield:

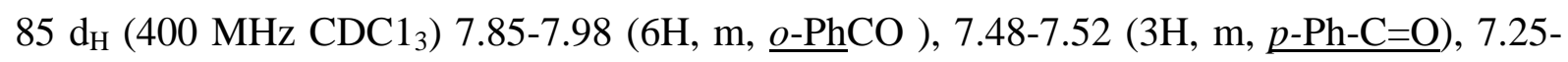
$7.42(6 \mathrm{H}, \mathrm{m}, \underline{m-\mathrm{PhCO}),} 7.18(2 \mathrm{H}, \mathrm{dd}, \mathrm{J} 7.5 \mathrm{~Hz}, \underline{o-\mathrm{PhOMe}}), 6.73(2 \mathrm{H}, \mathrm{dd}, J$ 7,1 Hz, $\underline{m-}$ PhOMe), 6.11 (1H, t, J 9.6 Hz, H-3), 5.64 (1H, t, J 9 Hz, H-4),5.29-5.54 (2H, m, H-1, H-2), 4.54 (2H, dd, J 11.6 Hz C$\left.\underline{H}_{2} \mathrm{PhOMe}\right), 4.23-4.19$ (1H, m, H-5), 3.72 (3H, s, PhOMe), 3.65$3.63(2 \mathrm{H}, \mathrm{m}, \mathrm{H}-6, \mathrm{H}-6), 3.49(3 \mathrm{H}, \mathrm{s}, \mathrm{OMe}) ; \mathrm{d}_{\mathrm{C}}\left(100.6 \mathrm{MHz}, \mathrm{CDCl}_{3}\right)$ 165.8, 161.2, 133.2, $129.9,129.4,128.4,128.2,113.7,97.2,73.3,72.2,70.71,69.5,68.9,68.1,55.6,55.2 . \mathrm{MS} \mathrm{m} / \mathrm{z}$ (\%) $649.6(80)[\mathrm{M}+\mathrm{Na}]^{+} .{ }^{1} \mathrm{H}$ and ${ }^{13} \mathrm{C}$ NMR spectra were identical with those in the literature ${ }^{8}$.

References:

8. Miranda, P. O.; Brouard, I.; Padron, J. I.; Bermejo J. Tetrahedron Letters, ” Ferric chloride: a mild and versatile reagent for the formation of 1,6-anhydro glucopyranoses", 2003, 44, 3931.

\section{Methyl-2,3-di-O-benzyl-6-O-tert-butylodimethylsilyl-4-O-(4-methoxybenzy)l- $\alpha$-D-}

glucopyranoside (4i) ${ }^{9}$ Yield: $84 \%$; colorless oil; $\mathrm{d}_{\mathrm{H}}\left(400 \mathrm{MHz} \mathrm{CDC1}_{3}\right)$ 7.33-7.26 (10H, m, benzyl), $7.10(2 \mathrm{H}, \mathrm{d}, J 8.4 \mathrm{~Hz}, \underline{o-\mathrm{Ph}}), 6.84(2 \mathrm{H}, \mathrm{d}, J 8.4 \mathrm{~Hz}, \underline{m-\mathrm{Ph}}), 5.03(1 \mathrm{H}, \mathrm{d}, \mathrm{J}=11.6$ $\left.\mathrm{Hz}, \underline{\mathrm{C}}_{2} \underline{\mathrm{Ph}}\right), 4.72\left(1 \mathrm{H}, \mathrm{d}, \mathrm{J}=11.6 \mathrm{~Hz}, \underline{\mathrm{H}}_{2} \mathrm{Ph}\right), 4.66\left(1 \mathrm{H}, \mathrm{d}, \mathrm{J}=12 \mathrm{~Hz}, \underline{\mathrm{C}}_{2} \underline{\mathrm{Ph}}\right), 4.55(1 \mathrm{H}, \mathrm{d}$, $\mathrm{J}=3.6 \mathrm{~Hz}, \mathrm{H}-1), 4.56\left(1 \mathrm{H}, \mathrm{d}, \mathrm{J}=12 \mathrm{~Hz}, \underline{\mathrm{H}}_{2} \mathrm{Ph}\right), 3.86-3.65\left(8 \mathrm{H}, \mathrm{m}, \underline{\mathrm{C}}_{2} \underline{\mathrm{Ph}}, \mathrm{PhOMe}, \mathrm{H}-2, \mathrm{H}-3\right.$, H-4) 3.53-5.52 (2H, m, H-5,H-6), 3.46 (1H, dd, J 4.0 Hz, J 9.6 Hz, H-6 ), 3.37 (3H s, OMe), 0.88, 0.05, $0.04\left(3 \mathrm{~s}, 15 \mathrm{H}, \mathrm{t}-\mathrm{BuMe}_{2} \mathrm{Si}\right) ; \mathrm{d}_{\mathrm{C}}\left(100.6 \mathrm{MHz}, \mathrm{CDCl}_{3}\right)$ 159.8,138.1, 129.2, 128.4, $128.2,127.8,127.3,97.5,81.7,80.8,77.3,76.7,75.0,73.2,62.6,53.0,55.9,25.9,18.1,-5.01$, 
-5.36.; ESI HRMS: Calcd for $\mathrm{C}_{35} \mathrm{H}_{48} \mathrm{O}_{7}{ }^{-} \mathrm{NaSi}$ : 631.3062; found: $\mathrm{m} / \mathrm{z} 631.3070[\mathrm{M}+\mathrm{Na}]^{+} .{ }^{1} \mathrm{H}$ , ${ }^{13} \mathrm{C}$ NMR and HMRS spectra were identical with those in the literature 9.

References:

9. Borsuk, K.; Kazimierski, A.; Solecka, J.; Urbańczyk-Lipkowska, Z.; Chmielewski, M. Carbohydrate Res. "Stereocontrolled formation of oxacephams from carbohydrates”, 2002, 337, 2005. 